Pak. j. sci. ind. res. Ser. B: biol. sci. 2021 64B(1) 49-54

\title{
Growth, Yield and Quality Response of Three Wheat Varieties to Foliar Spray of Micro Nutrients
}

\author{
Muhammad Tahir ${ }^{a}$, Muhammad Tahir Naveed ${ }^{a *}$ Aftab Ahmad Sheikh ${ }^{\mathrm{b}}$ and Rizwan Maqbool \\ ${ }^{a}$ Department of Agronomy, University of Agriculture, Faisalabad, Pakistan \\ ${ }^{b}$ Institute of Soil Chemistry and Environmental Sciences, Kala Shah Kaku, Pakistan
}

(received April 9, 2019; revised September 17, 2019; accepted September 19, 2019)

\begin{abstract}
A field experiment was carried out to test the result of foliar application of micro nutrients on growth, quality and yield of three wheat verities. The trial was performed at Agronomic research area, University of Agriculture Faisalabad. Wheat varieties Jouhar 2016, Ujala 2015 and Gold 2016 were sown. Combination of micro nutrient ( $\mathrm{Zn} 2 \%, \mathrm{~B} 0.5 \%, \mathrm{Fe} 1 \%, \mathrm{Mn} 1 \%$ and $\mathrm{Cu} 0.5 \%$ ) with different amounts (no spray, water spray, $1000 \mathrm{~mL} / \mathrm{ha}, 1250 \mathrm{~mL} / \mathrm{ha}$ and $1500 \mathrm{~mL} / \mathrm{ha}$ ) was sprayed at booting stage. By results of this study,it is concluded that foliar spray of micro nutrients has significant effect on growth, yield and quality parameters. Statistically highly significant effect of $1250 \mathrm{~mL} / \mathrm{ha}$ spray of micro nutrients mixture was observed on plant height $(105.33 \mathrm{~cm})$, grains/spike (48), grain yield $(5336.3 \mathrm{Kg} / \mathrm{ha})$, biological yield $(12829 \mathrm{Kg} / \mathrm{ha})$, carbohydrates (63.7\%) and protein (11.4\%) under $\mathrm{V}_{2} \mathrm{~F}_{4}$ (Ujala $2015+1250 \mathrm{~mL} /$ ha micro nutrients).
\end{abstract}

Keywords: wheat varieties, foliar application, $\mathrm{Zn}, \mathrm{Fe}, \mathrm{Mn}$ and $\mathrm{Cu}$

\section{Introduction}

Wheat is an important cereal crop of Pakistan. It is sown worldwide on wide range of different climatic conditions as compared to the other grain crops. Wheat is rich source of protein, vitamins, minerals and carbohydrates (Habib, 2009). Wheat contribution in diet is about 70-72\% (Ali et al., 2013; Habbasha et al., 2013). Micro nutrients deficiency in world population is more than 40\% (Faraji et al., 2014; Abbas et al., 2011). Growth, development and yield of wheat crop decreased due to deficiency of micro nutrients (Nadim et al., 2012; Cisse and Amar, 2000). In Pakistan about 1980 thousand hectares is wheat cultivated area and production is about 25.48 million tons. Agriculture's share in GDP is $18.9 \%$ and out of which $9.1 \%$ is contributed by wheat (Govt. of Pakistan, 2018). Different factors trigger yield losses like late sowing, non-judicious use of fertilizer, less irrigation, excess of weeds in fields and drought for long time. (Zeidan et al., 2010). These factors are responsible in various stages of crop and harmfully impact the crop yield. For various crops, micro and macro nutrients deficiencies have been examined (Hussain et al., 2006). To regulate different metabolic processes and yield, each micronutrient plays a vital role. Zinc, Boron, Iron, Copper, Manganese and Molybdenum are important for plant's optimum growth and development (Rawashdeh and Florin, 2015; Zain

*Author for correspondence; E-mail: mtahir187@yahoo.com et al., 2015). Boron is most essential for reproductive parts, cell multiplication, cell stabilization, carbohydrate utilization and formation of cell wall in plants (Biswas et al., 2015; Khan et al., 2010). Fe functions in chlorophyll biosynthesis,respiration, chloroplast development, carbohydrate productions, enzyme activation, biological redox system and act as oxygen carrier in nitrogen fixation (Rasul et al., 2015). Manganese is compulsory for metabolic reactions, accelerates chlorophyll biosynthesis, enzyme activation, electron transport, progresses immune system, improves uptake of $\mathrm{Ca}$ and $\mathrm{P}$ and assists in photosynthesis (Abbas et al., 2011). Copper performs numerous metabolic processes, aids in physiological redox processes, lignification and improves the uptake of $\mathrm{N}$ and interfaces with the other micro nutrients (Monreal et al., 2015; Habbasha et al., 2013). Increase in crop production cause the micro nutrients deficiencies (Fageria et al., 2002). Pakistani soils have deficiency of different micro nutrients such as boron deficiency is about 51-60\% (Rashid et al., 2002). Wheat production can be enhanced by enhancing the yield per unit area. Plants require proper amounts of macro nutrients and micro nutrients for better growth.

\section{Materials and Methods}

A field trial was conducted at Agronomic Farm University of Agriculture, Faisalabad during growing season 2016-17. Texture of soil was sandy loam. An 
experiment was laid out in randomized complete block design with factorial arrangements and three replications. Plot size was $6.0 \mathrm{~m}$ long x $2.25 \mathrm{~m}$ wide. Treatments were different wheat varieties: $V_{1}=$ Jouhar 2016; $\mathrm{V}_{2}=$ Ujala 2015; $\mathrm{V}_{3}=$ Gold 2016 and foliar applications: $\mathrm{F}_{1}=$ control(no spray); $\mathrm{F}_{2}=$ water spray; $\mathrm{F}_{3}=1000 \mathrm{~mL} /$ micro nutrients ( $\mathrm{Zn} 4.7 \%$, B $1.0 \%$, Fe 2\%, Mn 2\%, and $\mathrm{Cu} 3 \%) / \mathrm{ha} \mathrm{F}_{4}=1250 \mathrm{~mL}$ micro nutrients $(\mathrm{Zn} 4.7 \%$, $\mathrm{B} 1.0 \%$, Fe $2 \%$, Mn 2\%, and $\mathrm{Cu} 3 \%$ ) $/$ ha and $\mathrm{F}_{5}=1500$ $\mathrm{mL}$ micro nutrients(Zn 4.7\%, B 1.0\%, Fe 2\%, Mn 2\%, and $\mathrm{Cu} 3 \%$ )/ha. Only single dose of spray was applied at booting stage. Recorded data was analysed statistically by Fisher's analysis of variance technique and treatments means were compared by using least significant difference (LSD) test at 5\% level of probability (Steel et al., 1997).

\section{Results and Discussion}

Plant height (cm). The data showed that the plant height was significantly affected by varieties, foliar spray of micro nutrients and their interaction. The response of different wheat varieties and foliar application was found significant. The maximum plant height $(105.33 \mathrm{~cm})$ was obtained in treatment $\mathrm{V}_{1} \mathrm{~F}_{4}$ (Jouhar $2016+1250 \mathrm{~mL} / \mathrm{ha}$ micro nutrients). and the Minimum plant height $(94.33 \mathrm{~cm})$ was obtained in $\mathrm{V}_{3} \mathrm{~F}_{1}$ (Gold 2016 + Control), however, it is statistically at par with treatments $\mathrm{V}_{2} \mathrm{~F}_{1}$ (Ujala $2015+$ Control) and $\mathrm{V}_{3} \mathrm{~F}_{2}$ (Gold $2016+$ Water spray). The findings of present study are corelated with Khan et al. (2010) which stated increased plant height by the micro nutrient's spray applied at different stages. Hussain et al. (2005) and Nadim et al. (2012) also described the similar results.

Number of tillers. Numbers of total and productive tillers are statistically non-significant for different wheat varieties, foliar application and their interaction.

Number of grains per spike. Statistically a greater number of grains per spike (48) was found under $\mathrm{V}_{2} \mathrm{~F}_{4}$ (Ujala $2015+1250 \mathrm{~mL} /$ ha micro nutrients) followed by $\mathrm{V}_{1} \mathrm{~F}_{4}$ (Jouhar $2016+1250 \mathrm{~mL} / \mathrm{ha}$ micro nutrients) that produced 46 grains per spike. The lowest number of grains per spike (37) was in $\mathrm{V}_{1} \mathrm{~F}_{1}$ (Jouhar $2016+$ control) and $\mathrm{V}_{3} \mathrm{~F}_{1}$ (Gold $2016+$ control) that is statistically similar with $\mathrm{V}_{2} \mathrm{~F}_{1}$ (Ujala $2015+$ control), $\mathrm{V}_{1} \mathrm{~F}_{2}$ (Jouhar 2016 + water spray) and $\mathrm{V}_{3} \mathrm{~F}_{2}$ (Gold 2016 + water spray). Tahir et al. (2009) represented that foliage use of micro nutrients efficiently improved grains per spike. Micro nutrients mixture enhanced the grains per spike in wheat (Yaseen et al. 2011). Same results were described by Khan et al. (2010).

1000- grain weight (g). Treatment V2F4 (Ujala 2015 $+1250 \mathrm{~mL} / \mathrm{ha}$ micro nutrients) gained significantly maximum 1000-grain weight (49 g). However, the lowest 1000-grain weight $(41 \mathrm{~g})$ was found under the treatment $\mathrm{V}_{3} \mathrm{~F}_{1}$ (Gold 2016 + control) that is statistically at par with treatments $\mathrm{V}_{1} \mathrm{~F}_{1}$ (Jouhar 2016 + control) and $\mathrm{V}_{2} \mathrm{~F}_{1}$ (Ujala $2015+$ control). The findings are corelated with Yaseen et al. (2010). The results are also considered by Kassab et al. (2004) and Torun et al. (2001) which stated that thousand grain weight in case of wheat was significantly increased by foliar application zinc.

Grain yield (Kg/ha). The treatment V2F4 (Ujala 2015 $+1250 \mathrm{~mL} /$ ha micro nutrients) produced statistically highest grain yield $(5336.3 \mathrm{Kg} / \mathrm{ha})$ followed by $\mathrm{V}_{3} \mathrm{~F}_{4}$ (Gold 2016 $+1250 \mathrm{~mL} /$ ha micro nutrients). Whereas the lowest grain yield was showed under response of $\mathrm{V}_{3} \mathrm{~F}_{1}$ (Gold $2016+$ control) that is statistically same with treatments like as $\mathrm{V}_{1} \mathrm{~F}_{1}$ (Jouhar 2016 + control), $\mathrm{V}_{2} \mathrm{~F}_{1}$ (Ujala $2015+$ control) and $\mathrm{V}_{3} \mathrm{~F}_{2}$ (Gold $2016+$ qater spray). Yaseen et al. (2010) stated that foliar use of all the nutrients cause enhance $24-38 \%$ yield of the wheat crop.

Biological yield (Kg/ha). The treatment $\mathrm{V}_{2} \mathrm{~F}_{4}$ (Ujala $2015+1250 \mathrm{~mL} / \mathrm{ha}$ micro nutrients) produced maximum biological yield $(12829 \mathrm{Kg} / \mathrm{ha})$. The lowest biological yield $(9946 \mathrm{Kg} / \mathrm{ha})$ was accumulated in treatment $\mathrm{V}_{3} \mathrm{~F}_{1}$ (Gold $2016+$ control) that is statistical similar with treatments V1F1 (Jouhar 2016 + control), $\mathrm{V}_{2} \mathrm{~F}_{1}$ (Ujala $2015+$ control), $\mathrm{V}_{1} \mathrm{~F}_{2}$ (Jouhar $2016+$ water spray) and $\mathrm{V}_{3} \mathrm{~F}_{2}$ (Gold 2016 + water spray) which produced 10686 $\mathrm{Kg} / \mathrm{ha}, 10484 \mathrm{Kg} / \mathrm{ha}, 10583 \mathrm{Kg} / \mathrm{ha}$ and $10538 \mathrm{Kg} / \mathrm{ha}$ respectively. The conclusions are union with Khan et al. (2010) and Hussain et al. (2005) they stated that total biomass enhanced with the foliar nourishing of micro nutrients. The results are also in line with Esfandiari et al. (2016).

Straw yield (Kg/ha). Treatment $\mathrm{V}_{2} \mathrm{~F}_{4}$ (Ujala $2015+$ $1250 \mathrm{~mL} /$ ha micro nutrients) collected highest straw yield amount $(7492.7 \mathrm{Kg} / \mathrm{ha})$ that is statistically at par with treatments of $\mathrm{V}_{2} \mathrm{~F}_{3}$ (Ujala $2015+1000 \mathrm{~mL} / \mathrm{ha}$ micro nutrients), $\mathrm{V}_{3} \mathrm{~F}_{4}$ (Gold $2016+1250 \mathrm{~mL} / \mathrm{ha}$ ), $\mathrm{V}_{1} \mathrm{~F}_{4}$ (Jouhar $2016+1250 \mathrm{~mL} / \mathrm{ha}$ ), $\mathrm{V}_{2} \mathrm{~F}_{5}$ (Ujala $2015+1500$ $\mathrm{mL} / \mathrm{ha}$ ), $\mathrm{V}_{3} \mathrm{~F}_{3}$ (Gold $2016+1000 \mathrm{~mL} /$ ha micro nutrients) and $\mathrm{V}_{1} \mathrm{~F}_{3}$ (Jouhar $2016+1000 \mathrm{~mL} /$ ha micro nutrients). 
However, the lowest straw yield $(6222.7 \mathrm{Kg} / \mathrm{ha})$ was exhibited in treatment $\mathrm{V}_{3} \mathrm{~F}_{1}$ (Gold $2016+$ control) that is statistically same with treatments $\mathrm{V}_{1} \mathrm{~F}_{1}$ (Jouhar 2016 + control), $\mathrm{V}_{2} \mathrm{~F}_{1}$ (Ujala $2015+$ control), $\mathrm{V}_{1} \mathrm{~F}_{2}$ (Jouhar 2016 + water spray), $\mathrm{V}_{2} \mathrm{~F}_{2}$ (Ujala $2015+$ water spray), $\mathrm{V}_{3} \mathrm{~F}_{2}$ (Gold $2016+$ water spray), $\mathrm{V}_{1} \mathrm{~F}_{5}$ (Jouhar $2016+$ $1500 \mathrm{~mL} / \mathrm{ha}$ ) and $\mathrm{V}_{3} \mathrm{~F}_{5}$ (Gold $2016+1500 \mathrm{~mL} / \mathrm{ha}$ ). The findings of the current study are considered by ElGhamry et al. (2009) who documented that micronutrients application improved straw yield in wheat. The same results are also presented by Ananda and Patil (2005).

Harvest index (\%). Harvest index percent is the ratio of total biological yield to economic yield. Data revealed that regarding harvest index the maximum amount (41.65\%) was observed in treatment $\mathrm{V}_{2} \mathrm{~F}_{4}$ (Ujala 2015 $+1250 \mathrm{~mL} /$ ha micro nutrients) than all other treatments but this treatment effect was statistically not differ with $\mathrm{V}_{1} \mathrm{~F}_{4}$ (Jouhar $2016+1250 \mathrm{~mL} /$ ha micro nutrients), $\mathrm{V}_{3} \mathrm{~F}_{4}$ (Gold $2016+1250 \mathrm{~mL} /$ ha micro nutrients), $\mathrm{V}_{1} \mathrm{~F}_{3}$ (Jouhar
$2016+1000 \mathrm{~mL} /$ ha micro nutrients) and $\mathrm{V}_{3} \mathrm{~F}_{3}$ (Gold $2016+1000 \mathrm{~mL} / \mathrm{ha}$ micro nutrients). Whereas, the lowest harvest index percent (37.44\%) was counted in treatment $\mathrm{V}_{3} \mathrm{~F}_{1}$ (Gold $2016+$ control) that is statistical at par with $\mathrm{V}_{2} \mathrm{~F}_{1}$ (Ujala $2015+$ control), $\mathrm{V}_{1} \mathrm{~F}_{1}$ (Jouhar $2016+$ control), $\mathrm{V}_{1} \mathrm{~F}_{2}$ (Jouhar $2016+$ water spray), $\mathrm{V}_{2} \mathrm{~F}_{2}$ (Ujala $2015+$ water spray) and $\mathrm{V}_{2} \mathrm{~F}_{5}$ (Ujala $2015+$ $1500 \mathrm{~mL} / \mathrm{ha}$ micro nutrients). The findings of current study are correlated with Khan et al. (2010) as they have stated that harvest index enhanced with micro nutrients application. Same findings are also reported by Zain et al. (2015) too who stated that foliar use of micro nutrients increases the harvest index.

Protein contents (\%). Treatment $\mathrm{V}_{2} \mathrm{~F}_{4}$ (Ujala $2015+$ $1250 \mathrm{~mL} /$ ha micro nutrients) statistically highest protein contents $(11.4 \%)$. Minimum protein percentage $(9.0 \%)$ was recorded under treatment of $\mathrm{V}_{3} \mathrm{~F}_{1}$ (Gold $2016+$ control) that is statistically similar with treatment $\mathrm{V}_{1} \mathrm{~F}_{1}$ (Jouhar 2016 + control). Micro nutrients contributions in physiological procedures plants like amino acid

Table 1. Individual comparison of treatment means

\begin{tabular}{|c|c|c|c|c|c|}
\hline Treatments & Plant height $(\mathrm{cm})$ & No. of tillers $/ \mathrm{m}$ & $\begin{array}{l}\text { No. of productive } \\
\text { tillers } / \mathrm{m}^{2}\end{array}$ & $\begin{array}{l}\text { No. of grains } \\
\text { per Spike }\end{array}$ & $\begin{array}{l}\text { 1000-Seed } \\
\text { weight }\end{array}$ \\
\hline $\mathrm{V}_{1-J o u h a r-2016}$ & $102.80 \mathrm{~A}$ & 306.27 & $41.60 \mathrm{AB}$ & 301.80 & $45.20 \mathrm{AB}$ \\
\hline V 2 - Ujala-2015 & $98.73 \mathrm{~B}$ & 307.00 & $43.00 \mathrm{~A}$ & 304.27 & $45.86 \mathrm{~A}$ \\
\hline $\mathrm{V}_{3-}$ Gold-2016 & $96.47 \mathrm{C}$ & 306.53 & $41.20 \mathrm{~B}$ & 303.47 & $44.66 \mathrm{~B}$ \\
\hline LSD value at $5 \%$ & 0.82 & NS & NS & 1.48 & 0.72 \\
\hline $\mathrm{F}_{1-}$ Control & $97.00 \mathrm{C}$ & 306.33 & $37.33 \mathrm{D}$ & 300.22 & $41.66 \mathrm{E}$ \\
\hline $\mathrm{F}_{2}$-Water Spray & $98.00 \mathrm{C}$ & 306.22 & $39.66 \mathrm{C}$ & 303.89 & $44.44 \mathrm{D}$ \\
\hline $\mathrm{F}_{3}-1000 \mathrm{~mL}$ micro nutrients/ha & $99.56 \mathrm{~B}$ & 307.89 & $44.00 \mathrm{~B}$ & 305.00 & $46.44 \mathrm{~B}$ \\
\hline $\mathrm{F}_{4-} 1250 \mathrm{~mL}$ micro nutrients/ha & $102.22 \mathrm{~A}$ & 308.78 & $46.66 \mathrm{AB}$ & 305.78 & $48.22 \mathrm{~A}$ \\
\hline $\mathrm{F}_{5^{-}} 1500 \mathrm{~mL}$ micro nutrients/ha & $99.89 \mathrm{~B}$ & 303.78 & $46.67 \mathrm{~A}$ & 301.00 & $45.44 \mathrm{C}$ \\
\hline LSD value at $5 \%$ & 1.07 & NS & NS & 1.91 & 0.93 \\
\hline $\mathrm{V}_{1} \times \mathrm{F}_{1}$ & $101.33 \mathrm{bc}$ & 306.00 & $37.00 \mathrm{~g}$ & 293.00 & $41.66 \mathrm{fg}$ \\
\hline $\mathrm{V}_{1} \times \mathrm{F} 2$ & $101.67 \mathrm{~b}$ & 307.00 & $39.00 \mathrm{e}-\mathrm{g}$ & 305.00 & $45.00 \mathrm{e}$ \\
\hline $\mathrm{V}_{1} \times \mathrm{F} 3$ & $103.00 \mathrm{~b}$ & 307.00 & $44.00 \mathrm{~b}-\mathrm{d}$ & 306.00 & $46.33 \mathrm{c}-\mathrm{e}$ \\
\hline $\mathrm{V}_{1} \times \mathrm{F} 4$ & $105.33 \mathrm{a}$ & 307.00 & $46.00 \mathrm{ab}$ & 304.33 & $48.00 \mathrm{ab}$ \\
\hline $\mathrm{V}_{1} \times \mathrm{F} 5$ & $102.67 \mathrm{~b}$ & 304.00 & $42.00 \mathrm{c}-\mathrm{e}$ & 300.67 & $45.00 \mathrm{e}$ \\
\hline $\mathrm{V}_{2} \times \mathrm{F}_{1}$ & $95.33 \mathrm{fg}$ & 306.00 & $38.00 \mathrm{fg}$ & 303.33 & $42.33 \mathrm{fg}$ \\
\hline $\mathrm{V}_{2} \times \mathrm{F}_{2}$ & $97.33 \mathrm{e}$ & 306.00 & $41.00 \mathrm{~d}-\mathrm{f}$ & 303.67 & $45.33 \mathrm{e}$ \\
\hline $\mathrm{V}_{2} \times \mathrm{F}_{3}$ & $99.33 \mathrm{~d}$ & 307.33 & $45.00 \mathrm{a}-\mathrm{c}$ & 304.67 & $47.00 \mathrm{~b}-\mathrm{d}$ \\
\hline $\mathrm{V}_{2} \times \mathrm{F}_{4}$ & $102.00 \mathrm{~b}$ & 311.00 & $48.00 \mathrm{a}$ & 307.67 & $49.00 \mathrm{a}$ \\
\hline $\mathrm{V}_{3} \times \mathrm{F}_{5}$ & $99.67 \mathrm{~cd}$ & 304.67 & $43.00 \mathrm{~b}-\mathrm{d}$ & 302.00 & $45.66 \mathrm{de}$ \\
\hline $\mathrm{V}_{3} \times \mathrm{F}_{1}$ & $94.33 \mathrm{~g}$ & 308.67 & $37.00 \mathrm{~g}$ & 304.33 & $41.00 \mathrm{~g}$ \\
\hline $\mathrm{V}_{3} \times \mathrm{F}_{2}$ & $95.00 \mathrm{fg}$ & 305.67 & $39.00 \mathrm{e}-\mathrm{g}$ & 303.00 & $43.00 \mathrm{f}$ \\
\hline $\mathrm{V}_{3} \times \mathrm{F}_{3}$ & $96.3 \mathrm{ef}$ & 307.67 & $43.00 \mathrm{~b}-\mathrm{d}$ & 304.33 & $46.00 \mathrm{de}$ \\
\hline $\mathrm{V}_{3} \times \mathrm{F}_{4}$ & $99.33 \mathrm{~d}$ & 308.33 & $46.00 \mathrm{ab}$ & 305.33 & $47.66 \mathrm{a}-\mathrm{c}$ \\
\hline $\mathrm{V}_{3} \times \mathrm{F}_{5}$ & $97.33 \mathrm{e}$ & 302.67 & $41.00 \mathrm{~d}-\mathrm{f}$ & 300.33 & $45.66 \mathrm{de}$ \\
\hline LSD value at $5 \%$ & 1.85 & NS & NS & 3.31 & 1.60 \\
\hline
\end{tabular}


Table 2. Individual comparison of treatment means

\begin{tabular}{|c|c|c|c|c|c|c|}
\hline Treatments & $\begin{array}{l}\text { Grain yield } \\
(\mathrm{Kg} / \mathrm{ha})\end{array}$ & $\begin{array}{l}\text { Biological } \\
\text { Yield }(\mathrm{Kg} / \mathrm{ha})\end{array}$ & $\begin{array}{l}\text { Straw yield } \\
(\mathrm{Kg} / \mathrm{ha})\end{array}$ & $\begin{array}{l}\text { Harvest } \\
\text { Index }\end{array}$ & $\begin{array}{l}\text { Protein } \\
\text { content }(\%)\end{array}$ & $\begin{array}{l}\text { Carbohydrates } \\
(\%)\end{array}$ \\
\hline V1-Jouhar-2016 & $4424.9 \mathrm{AB}$ & $11145 \mathrm{~B}$ & $6719.6 \mathrm{~B}$ & 39.61 & $10.04 \mathrm{~B}$ & $62.12 \mathrm{~B}$ \\
\hline $\mathrm{V}_{2}$ - Ujala-2015 & $4555.0 \mathrm{~A}$ & $11624 \mathrm{~A}$ & $7069.4 \mathrm{~A}$ & 39.09 & $10.28 \mathrm{~A}$ & $62.36 \mathrm{~A}$ \\
\hline $\mathrm{V}_{3-}$ Gold-2016 & $4314.0 \mathrm{~B}$ & $11057 \mathrm{~B}$ & $6742.7 \mathrm{~B}$ & 38.93 & $9.98 \mathrm{~B}$ & $61.92 \mathrm{C}$ \\
\hline LSD value at $5 \%$ & 153.08 & 354.19 & 257.31 & NS & 0.11 & 596.26 \\
\hline $\mathrm{F}_{1}$-Control & $3848.7 \mathrm{E}$ & $10205 \mathrm{D}$ & $6356.8 \mathrm{C}$ & $37.71 \mathrm{D}$ & $9.13 \mathrm{E}$ & $60.76 \mathrm{E}$ \\
\hline $\mathrm{F}_{2}$-Water Spray & $4065.6 \mathrm{D}$ & $10664 \mathrm{C}$ & 6598.BC & $38.11 \mathrm{CD}$ & $9.54 \mathrm{D}$ & $61.20 \mathrm{D}$ \\
\hline $\mathrm{F}_{3-} 1000 \mathrm{~mL}$ micronutrients/ha & $4755.2 \mathrm{~B}$ & $11901 \mathrm{~B}$ & $7145.9 \mathrm{~A}$ & $39.97 \mathrm{~B}$ & $10.53 \mathrm{~B}$ & $62.96 \mathrm{~B}$ \\
\hline $\mathrm{F}_{4-} 1250 \mathrm{~mL}$ micronutrients/ha & $5171.1 \mathrm{~A}$ & $12498 \mathrm{~A}$ & $7326.6 \mathrm{~A}$ & $41.37 \mathrm{~A}$ & $11.03 \mathrm{~A}$ & $63.40 \mathrm{~A}$ \\
\hline $\mathrm{F}_{5-} 1500 \mathrm{~mL}$ micronutrients/ha & $4316.0 \mathrm{C}$ & $11108 \mathrm{C}$ & $6792.0 \mathrm{~B}$ & $38.89 \mathrm{BC}$ & $10.26 \mathrm{C}$ & $62.33 \mathrm{C}$ \\
\hline LSD value at $5 \%$ & 197.62 & 457.26 & 332.18 & 1.13 & 0.14 & 769.76 \\
\hline $\mathrm{V}_{1} \times \mathrm{F}_{1}$ & $3876.0 \mathrm{hi}$ & $10186 \mathrm{de}$ & $6310.0 \mathrm{e}$ & $38.06 \mathrm{ef}$ & $9.10 \mathrm{hi}$ & $60.80 \mathrm{gh}$ \\
\hline $\mathrm{V}_{1} \times \mathrm{F}_{2}$ & $4070.0 \mathrm{gh}$ & $10583 \mathrm{de}$ & $6537.7 \mathrm{de}$ & 38.43 ef & $9.40 \mathrm{fg}$ & $61.10 \mathrm{fg}$ \\
\hline $\mathrm{V}_{1} \times \mathrm{F}_{3}$ & $4745.0 \mathrm{c}-\mathrm{e}$ & $11713 \mathrm{~b}$ & 6967.7 a-d & $40.54 \mathrm{a}-\mathrm{d}$ & $10.50 \mathrm{~cd}$ & $63.00 \mathrm{~cd}$ \\
\hline $\mathrm{V}_{1} \times \mathrm{F}_{4}$ & $5170.3 \mathrm{ab}$ & $12411 \mathrm{ab}$ & $7240.7 \mathrm{ab}$ & $41.57 \mathrm{ab}$ & $10.90 \mathrm{~b}$ & $63.40 \mathrm{ab}$ \\
\hline $\mathrm{V}_{1} \times \mathrm{F}_{5}$ & $4263.3 \mathrm{fg}$ & $10830 \mathrm{~d}$ & $6567.0 \mathrm{c}-\mathrm{e}$ & $39.38 \mathrm{c}-\mathrm{f}$ & $10.30 \mathrm{de}$ & $62.30 \mathrm{e}$ \\
\hline $\mathrm{V}_{2} \times \mathrm{F}_{1}$ & $3946.7 \mathrm{~g}-\mathrm{i}$ & 10484 de & $6512.7 \mathrm{de}$ & $37.65 \mathrm{f}$ & $9.30 \mathrm{gh}$ & $60.90 \mathrm{gh}$ \\
\hline $\mathrm{V}_{2} \times \mathrm{F}_{2}$ & $4113.3 \mathrm{gh}$ & $10870 \mathrm{~cd}$ & $6756.7 \mathrm{~b}-\mathrm{e}$ & 37.84 ef & $9.63 \mathrm{f}$ & $61.40 \mathrm{f}$ \\
\hline $\mathrm{V}_{2} \times \mathrm{F}_{3}$ & $4884.0 \mathrm{~b}-\mathrm{d}$ & $12306 \mathrm{ab}$ & $7422.3 \mathrm{a}$ & $39.689 \mathrm{~b}-\mathrm{e}$ & $10.70 \mathrm{bc}$ & $63.10 \mathrm{bc}$ \\
\hline $\mathrm{V}_{2} \times \mathrm{F}_{4}$ & $5336.3 \mathrm{a}$ & 12829 a & $7492.7 \mathrm{a}$ & $41.65 \mathrm{a}$ & $11.40 \mathrm{a}$ & $63.70 \mathrm{a}$ \\
\hline $\mathrm{V}_{3} \times \mathrm{F}_{5}$ & 4494.7 ef & $11632 \mathrm{bc}$ & $7137.7 \mathrm{a}-\mathrm{c}$ & $38.70 \mathrm{~d}-\mathrm{f}$ & $10.40 \mathrm{~d}$ & $62.70 \mathrm{~d}$ \\
\hline $\mathrm{V}_{3} \times \mathrm{F}_{1}$ & $3723.3 \mathrm{i}$ & 9946 e & $6222.7 \mathrm{e}$ & $37.44 \mathrm{f}$ & $9.00 \mathrm{i}$ & $60.60 \mathrm{~h}$ \\
\hline $\mathrm{V}_{3} \times \mathrm{F}_{2}$ & $4013.3 \mathrm{gi}$ & $10538 \mathrm{de}$ & $6524.7 \mathrm{de}$ & 38.07 ef & $9.60 \mathrm{f}$ & $61.10 \mathrm{fg}$ \\
\hline $\mathrm{V}_{3} \times \mathrm{F}_{3}$ & $4636.7 \mathrm{de}$ & $11684 \mathrm{~b}$ & 7047.7 a-d & $40.544 \mathrm{a}-\mathrm{d}$ & $10.40 \mathrm{~d}$ & $62.80 \mathrm{~cd}$ \\
\hline $\mathrm{V}_{3} \times \mathrm{F}_{4}$ & $5006.7 \mathrm{a}-\mathrm{c}$ & $12253 \mathrm{ab}$ & $7246.3 \mathrm{ab}$ & $40.88 \mathrm{a}-\mathrm{c}$ & $10.80 \mathrm{~b}$ & $63.10 \mathrm{bc}$ \\
\hline $\mathrm{V}_{3} \times \mathrm{F}_{5}$ & $4190.0 \mathrm{f}-\mathrm{h}$ & $10862 \mathrm{~cd}$ & $6672.3 \mathrm{~b}-\mathrm{e}$ & 38.58 ef & $10.10 \mathrm{e}$ & $62.00 \mathrm{e}$ \\
\hline LSD value at $5 \%$ & 342.29 & 792.00 & 575.36 & 0.87 & 0.25 & 1333.3 \\
\hline
\end{tabular}

biosynthesis, activation of enzymes, and starch utilization, improved the accumulation of assimilates in seeds, which results in more protein contents in grain (Rasul et al., 2015; Khan et al., 2010).

Carbohydrate concentration (\%). Carbohydrate concentration revealed that statistically significant effect of foliar response of wheat varieties on concentration of carbohydrates. Maximum concentration $(63.7 \%)$ was found in treatment $\mathrm{V}_{2} \mathrm{~F}_{4}$ (Ujala $2015+1250 \mathrm{~mL} / \mathrm{ha}$ micro nutrients) followed by $\mathrm{V}_{3} \mathrm{~F}_{4}$ (Gold $2016+1250$ $\mathrm{mL} / \mathrm{ha}$ micro nutrients) that is obtained (63.1\%) carbohydrates. On the other hand, the statistically lowest carbohydrates concentration was gathered under treatment $\mathrm{V}_{3} \mathrm{~F}_{1}$ (Gold $2016+$ control) that is statistically at par with treatments $\mathrm{V}_{1} \mathrm{~F}_{1}$ (Jouhar $2106+$ control) and $\mathrm{V}_{2} \mathrm{~F}_{1}$ (Ujala 2015). The micro nutrient plays a vital role in several carbohydrate production, metabolic reactions, nitrogen fixation acts as an oxygen carrier, membrane integrity, starch utilization, in enzyme system acts as a co-factor and phytochrome activities (Monreal et al., 2015; Bameri et al., 2012).

\section{Conclusion}

This study concluded that foliar application of micro nutrients mixture (@1250 mL/ha) on wheat varieties produced higher yield and better quality of grains.

Conflict of Interest. The authors declare no conflict of interest

\section{References}

Abbas, G., Khan, M.Q., Khan, M.J., Tahir, M., Ishaque, M., Hussain, F. 2011. Nutrient uptake, growth and yield of wheat (Triticum aestivum L.) as affected by manganese application. Pakistan Journal of Botany, 43: 607-616.

Ali, A., Ali, N., Ullah, N., Ullah, F., Adnan, M., Swati, Z.A. 2013. Effect of drought stress on the physiology and yield of the Pakistani wheat 
germplasms. International Journal of Advancements in Research Technology, 2: 419-430.

Ananda, N., Patil, B.N. 2005. Effect of micro nutrients ( $\mathrm{Zn}$ and $\mathrm{Fe}$ ) and time of nitrogen application on growth and yield of durum wheat. Karnataka Journal Agriculture Science, 18: 604-608.

Bameri, M., Abdolshahi, R., Nejad, G.M., Yousefi, K., Tabatabaie, S.M. 2012. Effect of different microelement treatment on wheat (Triticum aestivum L.) growth and yield. International Research Journal of Applieed and Basic Sciences, 3: 219-223.

Biswas, A., Mukhopadhyay, D., Biswas, A. 2015. Effect of soil zinc and boron on the yield and uptake of wheat in an acid soil of west Bengal, India. International Journal of Plant and Soil Sciences, 6: 203217.

Cisse, L., Amar, B. 2000. The importance of phosphatic fertilizer for increased crop in developing countries, In proceedings of the AFA $6^{\text {th }}$ International Annual Conference, Cairo, Egypt.

El-Ghamry, A.M., Abd El-Hamid, A.M., Mosa, A.A., 2009. Effect of farmyard manure and foliar application of micro nutrients on yield characteristics of wheat grown on salt affected soil. American Eurasian Journal of Agriculture and Environmental Sciences, 5: 460-465.

Esfandiari, E., Abdoli, M., Mousavi, S.B., Sadeghzadeh, B. 2016. Impact of foliar zinc application on agronomic traits and grain quality parameters of wheat grown in zinc deficient soil. Indian Journal of Plant Physiology, 21: 263-270.

Fageria, N.K., Balinger, V.C., Clark, R.B. 2002. Micronutrients in crop production. Advances in Agronomy, 77: 185-268.

Faraji, H., Moradi, A., Jahanbin, S., Rahimi, A. 2014. Studying the effect of nutrient fertilizers on agronomical and biological yield of wheat $(\mathrm{CV}$ Alvand). Annals of Biology Research, 5: 96-98.

Govt. of Pakistan. 2018. Pakistan Economic Survey 2017-18. Ministry of finance, Islamabad. 2: 27-28.

Habbasha, S.F.E., Tawfik, M.M., El Kramany, M.F. 2013. Comparative efficacy of different biochemical foliar applications on growth, yield and yield attributes of some wheat cultivars. World Journal of Agricultue Science, 9: 345-353.

Habib, M. 2009. Effect of foliar application of $\mathrm{Zn}$ and Fe on wheat yield and quality. African Journal of Biotechnology, 8: 6795-6798.
Hussain, M.Z., Rehman, N., Khan, M.A., Roohullah, Ahmed, S.R. 2006. Micro nutrients status of Bannubasen soils. Sarhad Journal of Agriculture 22: 283285.

Hussain, N., Khan, M.A., Javed, M.A. 2005. Effect of foliar application of plant micro nutrient mixture on growth and yield of wheat (Triticum aestivum L.). Pakistan Journal of Biological Research, 8: 1096-1099.

Kassab, O.M., Zeing, H.A.E., Ibrahim, M.M. 2004. Effect of water deficient and micro nutrients foliar application on the productivity of wheat plants. Minufiya Journal of Agriculture Research, 29: 925 932.953-956.

Khan, M.B., Farooq, M., Hussain, M., Shahnawaz, M., Shabir, G. 2010. Foliar application of micro nutrients improves the wheat yield and net economic return. International Journal of Agriculture Policy and Biology, 12:

Monreal, C.M., DeRosa, M., Mallubhotla, S.C., Bindraban, P.S., Dimkpa, C. 2015. The Application of Nanotechnology for Micronutrients in Soil-Plant Systems. volume 3, pp1-5, Virtual Fertilizer Research Center, Washington, D.C., USA.

Nadim, M.A., Awan, I.U., Baloch, M.S., Khan, E.A., Naveed, K., Khan, M.A. 2012. Response of wheat (Triticum aestivum L.) to different micro nutrients and their application methods. Journal of Animal and Plant Sciences, 22: 113-119.

Rashid, A., Rafique, E., Ryan, J. 2002. Establishment and management of boron deficiency in crops in Pakistan. A country report. p: 339-48. In: Boron in Plant and Animal Nutrition. H.E. Goldbach, B. Rerkasem, M. Wimmer, P.H. Brown, M. Thellier and R.W. Bell (eds.). Kluwer Academic Publication, New York, USA.

Rasul, G.A.M., Salam, S.M., Rashid, B.R. 2015. Effect of iron application to calcareous soil on growth and yield of wheat in Sulaimani, Governorate-KurdistanIraq. American Eurasian Journal of Agriculture and Environmental Sciences, 15 : 1552-1555.

Rawashdeh, H.M., Florin, S. 2015. Foliar application with iron as a vital factor of wheat crop growth, yield quantity and quality: a review. International Journal of Agriculture Policy and Research, 3: 368-376.

Steel, R.G.D., Torrie, J.H., Dickey, D.A. 1997. Principles and Procedures of Statistics: A Biometrical 
Approach. $3^{\text {rd }}$ ed. pp 400-428. McGraw Hill Book Co. Inc., New York. USA.

Tahir, M., Tanveer, A., Shah,T.H., Fiaz, N., Wasaya, A. 2009. Yield response of wheat (Triticum aestivum L.) to boron application at different growth stages. Pakistan Journal Life Society Science, 7: 39-42.

Torun, A., ltekin, I.G.A., Kalayci, M., Yilmaz, A., Eker, S., Cakmak, I. 2001. Effects of zinc fertilization on grain yield and shoot concentrations of zinc, boron and phosphorus of 7425 wheat cultivars grown on a zinc-deficient and boron-toxic soil. Journal of Plant Nutrition, 2: 1817-1829.

Yaseen, A., Abou El-Nour, E.A.A., Shedeed, S. 2010. Response of wheat to foliar spray with urea and micronutrients. Journal of American Sciences, 6: 14-22.
Yaseen, M., Ahmed, W., Arshad, M., Ali., Q. 2011. Response of wheat (Triticum aestivum L.) to foliar feeding of micro nutrients. International Journal for Agro Veterinary and Medical Sciences, 5: 209220.

Zain, M., Khan, I., Qadri, R.W.K., Ashraf, U., Hussain, S., Minhas, S., Siddique, A., Jahangir, M.M., Bashir, M. 2015. Foliar application of micro nutrients enhances wheat growth, yield and related attributes. American Journal of Plant Sciences, 6: 864-869.

Zeidan, M.S., Mohamed, M.F., Hamouda, H.A. 2010. Effect of foliar fertilization of $\mathrm{Fe}, \mathrm{Mn}$ and $\mathrm{Zn}$ on wheat yield and quality in low sandy soils fertility. World Journal of Agriculture Sciences, 6: 696699. 